\title{
Education À L'entrepreneuriat Et Propension À Entreprendre En Contexte De Formation Professionnelle En Côte d'Ivoire
}

\author{
Benie Hillarion \\ Assistant d'Université au Département des Sciences de l'éducation, \\ Institut Pédagogique National de l'Enseignement Technique et Professionnel \\ (IPNETP), Côte d'Ivoire \\ Soungari Yeo \\ Assistant d'Université à l'Institut de Recherches, d'Etudes et \\ d'Expérimentation en Pédagogie (IREEP), \\ Université Félix Houphouët Boigny, Côte d'Ivoire
}

Doi: 10.19044/esj.2017.v13n28p335 URL:http://dx.doi.org/10.19044/esj.2017.v13n28p335

\begin{abstract}
Entrepreneurship education was introduced as a formal discipline in vocational training programs in the late 1990s. This study reflects on the relevance of this education to entrepreneurship in terms of the persistence of unemployment and the underemployment of graduates. Its general objective is to analyze the influence of the satisfaction of the training expectations of the trainees on their propensity to undertake. The survey was carried out at the Yopougon Business Professional High School and the Riviera Professional Hotel School. The instrument used to capture the level of satisfaction of trainees' expectations and their entrepreneurial propensity is a questionnaire with scales of measurement with three categories of response. The results establish a growing monotonous relationship between the satisfaction of the trainees expectations and their propensity to undertake. These results also indicate that satisfying three of the expectations expressed by trainees has a significant influence on their propensity to undertake. Entrepreneurship education is an effective strategy for the social construction of vocational integration through entrepreneurship among trainees in vocational training.
\end{abstract}

Keywords: Entrepreneurship education, occupational integration, Propensity to undertake, Relevance of the training 


\section{Resume}

L'enseignement de l'entrepreneuriat a été implanté comme une discipline officielle dans les programmes des établissements de formation professionnelle à la fin des années 1990. La présente étude mène une réflexion sur la pertinence de cette éducation à l'entrepreneuriat au regard de la persistance du chômage et du sous-emploi des diplômés. Elle a pour objectif général d'analyser l'influence de la satisfaction des attentes en formation des stagiaires sur leur propension à entreprendre. L'enquête a été réalisée au lycée professionnel commercial de Yopougon et au lycée professionnel hôtelier de la Riviera. L'instrument utilisé, pour saisir le niveau de satisfaction des attentes des stagiaires et leur propension entrepreneuriale, est un questionnaire comportant des échelles de mesure à trois catégories de réponse. Les résultats établissent une relation monotone croissante entre la satisfaction des attentes des stagiaires et leur propension à entreprendre. Ces résultats précisent également que la satisfaction de trois des attentes exprimées par les stagiaires a une influence significative sur leur propension à entreprendre. L'éducation à l'entrepreneuriat apparaît comme une stratégie efficace de construction sociale de l'insertion professionnelle par l'entrepreneuriat chez les stagiaires en formation professionnelle.

Mots-clés : Education à l'entrepreneuriat, insertion professionnelle, propension à entreprendre, pertinence de la formation

\section{Introduction}

La Côte d'Ivoire a opté, au lendemain de son indépendance en 1960, pour un capitalisme d'Etat. L'Etat était le principal acteur économique et sa politique industrielle était orientée vers les grandes unités ayant une grande capacité d'absorption de la main d'œuvre. La politique sociale de l'emploi de l'Etat consistait à offrir un emploi moderne à tous les demandeurs. Cette politique va progressivement imposer le «travail salarié » comme le modèle de travail de référence (Bazin et Gnabéli, 1997). Au cours de la décennie 70 - 80, le pays connait des difficultés économiques et financières « dues à la grande sécheresse de 1972-1973, aux chocs pétroliers de 1973 et 1979 et à l'importante chute des cours mondiaux du café et du cacao en 1979 » (Kouadio, 1998, p.1). L'Etat ne peut plus employer tous les demandeurs d'emploi notamment les diplômés. Pour remédier à l'amplification du chômage qui s'en suit, l'Etat ivoirien va engager des réformes aussi bien au niveau du marché du travail qu'au niveau du secteur éducatif. La loi de réforme de l'enseignement de 1995 et les plans nationaux de l'emploi (PNE) initiés à partir de 1991 vont faire la promotion de l'entrepreneuriat comme un mode alternatif d'insertion socioprofessionnelle des jeunes. En dépit de ces initiatives, l'évaluation des politiques de l'emploi montre que «les 
résultats de l'exécution du PNE de 1995, tout comme ceux de 1991 n'ont pas été satisfaisants [...] En effet, aucun des programmes contenus dans le PNE n'a pu atteindre les résultats escomptés »(OIT/PNUD, 2000, p 18-19).

Dans le cadre de la réforme du système éducatif en général et de celui de l'enseignement technique et de la formation professionnelle en particulier, l'enseignement de l'entrepreneuriat a été intégré comme une discipline officielle dans les programmes de formation à la fin des années 1990. A partir de 2009, un partenariat dénommé « Ecole - Entreprise » est également initié par le gouvernement en vue d'une meilleure adéquation entre la formation des diplômés et les opportunités d'emplois disponibles. Dans cette dynamique de réforme, "des modules de formation en entrepreneuriat ont été développés et déployés dans la formation pour promouvoir la culture entrepreneuriale, sans oublier le renforcement des mécanismes de mise en stage des élèves et étudiants en entreprise, de même que la promotion des dispositifs d'insertion professionnelle, à travers la mise en place et l'opérationnalisation d'un dispositif d'aide à l'insertion " (Koné, Koffi et Ehui, 2016, p. 78). L'implantation de cette nouvelle discipline, dans le dispositif de formation professionnelle, a exigé la formation de formateurs en entrepreneuriat à l'Institut Pédagogique National de l'Enseignement Technique et Professionnel (IPNETP) et la reconversion de certains enseignants des disciplines telles que « Economie » ou « Gestion commerciale ». Les formateurs en entrepreneuriat sont des enseignants recrutés par la fonction publique par voie de concours. Ils sont formés pendant deux (2) années sur des contenus relatifs à l'entrepreneuriat et aux pratiques pédagogiques à l'IPNETP. Ils effectuent, pendant leur formation, des micro-enseignements, des simulations et des stages pratiques dans des établissements d'enseignement technique et de formation professionnelle. Ils forment les stagiaires, dans les établissements, avec quasiment le même programme de formation. L'éducation à l'entrepreneuriat vise essentiellement l'atteinte de cinq (5) objectifs pédagogiques, à savoir, se situer au regard de l'entrepreneuriat ou la sensibilisation à la culture entrepreneuriale; décrire les étapes de la constitution d'une entreprise individuelle et d'une société ; décrire les principales étapes d'élaboration d'un projet d'entreprise ; expliquer la structure d'un plan d'affaires et monter un projet de création d'une entreprise. Ces objectifs pédagogiques de la formation en entrepreneuriat sont prévus pour être atteints avant la fin du cycle secondaire dans les lycées professionnels.

Après la crise militaro-politique de 2002 à 2011, soldée par une grave crise postélectorale qui a fait plus de 3000 morts (Regroupement des acteurs ivoiriens des droits humains, 2014), la Côte d'Ivoire a amorcé sa reconstruction. Les autorités politiques ivoiriennes ont engagé la relance de l'économie nationale en réalisant d'importants investissements consignés 
dans le plan national de développement (PND) de la période 2012 - 2015 et celui de la période 2016 - 2020. L'objectif de ces plans est de favoriser l'émergence de la Côte d'Ivoire à l'horizon 2020. Cette dynamique de développement économique de la Côte d'Ivoire est associée à une politique de réduction du chômage des jeunes en général et des diplômés en particulier. La politique de l'emploi en Côte d'Ivoire était gérée par plusieurs agences publiques d'insertion professionnelle (Agence d'Etudes et de Promotion de l'Emploi, Fonds National de la Jeunesse, Fonds sectoriels liés à l'insertion, etc.). Ces agences avaient pour missions d'assurer notamment l'accueil, l'information et l'orientation des jeunes demandeurs d'emploi; d'apporter un appui aux porteurs d'initiatives potentiellement créatrices d'emplois pour les jeunes; favoriser l'accès des jeunes porteurs de projets au crédit et promouvoir le dialogue avec les partenaires en faveur des populations cibles.

En dépit de ces initiatives, le chômage des diplômés demeure important et l'initiative privée reste faible chez ces derniers. En effet, selon le Ministère d'Etat, Ministère de l'Emploi, des Affaires Sociales et de la Formation Professionnelle (2014a), les 91\% des actifs sont occupés dans le secteur informel, caractérisé par des emplois précaires, de faibles productivités et de faibles rémunérations. Les statistiques du Ministère d'Etat, Ministère de 1'Emploi, des Affaires Sociales et de la Formation Professionnelle (2014b) indiquent que le taux de chômage de la population active est estimé à $6,7 \%$ en 2014 contre $8,7 \%$ en 2012. Au sein des jeunes de 14-24 ans, le taux de chômage est de 10,1\% en 2014 contre 12,8\% en 2012. Celui des 14-35 ans est de 8,6\% 2014 contre 11,4\% en 2012. Ces statistiques précisent que la proportion de chômeurs découragés est de 42,6\% en 2014 contre 41,8\% en 2012. Selon le Fonds Africain de Développement (2013), le taux de chômage en 2012 augmente avec le diplôme en Côte d'Ivoire. Ainsi, les statistiques indiquent -elles $38,5 \%$ de taux de chômage des diplômés de l'enseignement supérieur, $24 \%$ chez ceux de l'enseignement secondaire et $23,2 \%$ chez ceux de l'enseignement technique et de la formation professionnelle. Une étude récente du Secrétariat d'Etat chargé de l'enseignement technique et de la formation professionnelle (2017) a été réalisée auprès de $88,60 \%$ des diplômés de l'année 2016 en vue de suivre l'évolution de ceux-ci après leur sortie de l'école. Les résultats indiquent que $27,21 \%$ des répondants sont retournés aux études et $72,79 \%$ sont sur le marché du travail. Parmi les diplômés sur le marché du travail, les 14,43\% sont occupés dont $09,76 \%$ par l'entrepreneuriat et 36,98\% par un stage. En revanche, $85,5 \%$ des diplômés sur le marché du travail sont sans emploi. En l'absence d'études relatives à l'évaluation de l'implantation de l'éducation à l'entrepreneuriat, les données sur l'emploi suscitent des interrogations sur la problématique de l'insertion professionnelle des diplômés en Côte d'Ivoire. 
En particulier, ces données interrogent sur la pertinence de l'éducation à l'entrepreneuriat dans les établissements de formation professionnelle en Côte d'Ivoire. En d'autres termes, quelle est l'influence de l'éducation à l'entrepreneuriat sur la propension à entreprendre des diplômés des établissements de formation professionnelle?

\section{Cadre théorique}

Cette rubrique débute par une définition des concepts de pertinence de la formation, d'insertion professionnelle et d'intention entrepreneuriale.

\section{Pertinence de la formation}

La pertinence se définit comme le lien de conformité entre les objectifs de formation visés par l'établissement et les besoins de formation auxquels il doit répondre (Plante et Bouchard, 1998). L'évaluation de la pertinence de la formation consiste à comparer les objectifs de la formation avec les attentes des étudiants d'une part, et avec les besoins du marché de l'emploi d'autre part. Elle suppose selon Cros (2010) que l'apprenant est mis au centre des actions de formation de sorte que ce dernier en tire une plusvalue. La pertinence peut être évaluée en début, en cours ou en fin de formation. Dans le cas particulier où elle est évaluée au terme de la formation, elle vise essentiellement à analyser l'impact de la formation (Gérard, 2003).

\section{Insertion professionnelle}

Le concept d'insertion professionnelle a évolué dans la littérature. Il était appréhendé de manière linéaire comme l'accès à un emploi ou une transition vers le marché du travail (Trottier, 2000). Il est désormais perçu de manière quasi unanime comme un processus inscrit dans la durée, qui concerne des personnes n'ayant jamais appartenu à la population active et vise le développement de la qualification professionnelle de ces personnes afin d'accéder à une position stabilisée dans le système d'emploi (Drolet, Monette et Pelletier, 1996 ; Trottier, op. cit.; Vernières, 1997). Selon Alves (2005) par contre, l'insertion professionnelle est le résultat de l'interaction entre le milieu scolaire (ou de socialisation), le milieu professionnel et l'individu. Les facteurs influençant l'insertion professionnelle sont généralement le genre, l'âge, l'origine sociale, le statut professionnel des parents, les stratégies d'insertion, les représentations et pratiques de l'insertion, etc. (Aka, 2010 ; Diro, 2010; Koné, 2010). Ces facteurs sont d'ordre démographique, économique, éducatif, technologique, social et politique (Laflamme, 1984 ; Kane, Sy, Massing et Liboudou, 2014). Le présent travail retient la définition proposée par Alves (op. cit.) car elle permet de saisir une spécificité de la formation professionnelle notamment 
l'alternance de la formation des stagiaires entre milieu scolaire et milieu professionnel. Cette définition a l'avantage de permettre l'analyse du processus d'insertion professionnelle des diplômés avant la fin de leur étude.

\section{Propension entrepreneuriale}

L'entrepreneuriat est défini par la juxtaposition de ses quatre (4) conceptions que sont l'innovation (Verstraete et Fayolle, 2005 ; Schumpeter, 1935), la création d'opportunité (Lévy-Tadjine, Chelly et Paturel, 2006 ; Shane et Venkataraman, 2000), la création d'organisation (Gartner, 1988) et la création de valeur (Bruyat, 1993). L'étude retient, dans cette perspective, la définition de Laviolette et Loue (2006, p. 4) qui conçoivent l'entrepreneuriat comme "une dynamique de création et d'exploitation d'une opportunité d'affaires par un ou plusieurs individu(s) via la création de nouvelles organisations à des fins de création de valeur ». La valeur visée dans cette définition peut être d'ordre économique ou social. Car, "l'entrepreneuriat correspond tout à la fois à la volonté de combattre le chômage et la possibilité de trouver un équilibre satisfaisant entre le travail quotidien et l'adéquation de ses techniques et de ses capacités professionnelles » (Parmentier, 2015, p. 21).

Le processus de création d'une entreprise est constitué, selon Tounès (2006), de quatre phases que sont la propension, l'intention, la décision et l'acte de création. Dans le cadre du présent travail, la propension et l'intention sont considérées comme des notions similaires. La propension entrepreneuriale est alors définie comme le degré d'engagement dans l'action de créer une entreprise.

\section{Recension des écrits}

L'insertion professionnelle se concrétise soit par le salariat, soit par l'entrepreneuriat (Kolvereid, 1996). L'accès à un emploi dépend, pour une grande part, de l'étendue et de la qualité du réseau de relation ou du capital social des individus (Alves, op. cit. ; Brou, 2016 ; Koné, Koffi et Ehui, op. cit). Le capital social est particulièrement mobilisé pour l'insertion professionnelle par le salariat considéré comme le modèle de travail de référence par la population (Bazin et Gnabéli, op. cit.). Le travail salarié constituait, en ce sens, le socle de l'identité des hommes, en particulier, dans la sphère publique et la source de leur légitimité en tant que époux et père dans la sphère familiale (Fortino, 2009). "Le travail salarié s'est donc affirmé comme la matrice structurante des rapports sociaux, comme facteur d'intégration à la société globale. Il procure un statut à l'individu et une place dans la société; il fournit une identité sociale ». (Parmentier, op. cit., pp. 21). 
Les orientations et les pratiques du milieu scolaire relatives à l'insertion professionnelle influencent la façon dont les diplômés abordent l'insertion professionnelle au terme de leur formation (Alves, op. cit.). La finalité de l'éducation à l'entrepreneuriat serait de déconstruire le modèle du travail salarié en légitimant l'insertion professionnelle des diplômés par l'entrepreneuriat (Benié, 2012a). A cet effet, des ressources idéologiques, sociales et symboliques qui fonctionnent comme des références idéologiques favorables à l'insertion et au maintien dans l'entrepreneuriat sont mobilisées (Gnabéli et Lognon, 2010). La création d'entreprise se présente, dès lors, comme une opportunité pour construire son propre univers professionnel (Parmentier, op. cit.). L'éducation à l'entrepreneuriat vise justement à sensibiliser à l'alternative de carrière que représente la création d'entreprise en inculquant aux individus un état d'esprit et des capacités entrepreneuriales (Kallel, 2005 ; Verstraete, 1998). L'activité pédagogique la plus répandue dans l'éducation à l'entrepreneuriat est la microentreprise. Elle permet aux apprenants de créer et de gérer leur propre entreprise dans le cadre de leur parcours scolaire (Pepin, 2011a). L'expérience montre que l'éducation à l'entrepreneuriat modifie positivement l'attitude des apprenants vis-à-vis de la création d'entreprise et leur confiance en leur capacité entrepreneuriale (Boissin, Chollet et Emin, 2005 ; Pepin, 2011b). Le développement des capacités entrepreneuriales apparait dans cette éducation comme une variable déterminante pour l'insertion professionnelle par l'entrepreneuriat. Le modèle de la formation de l'événement entrepreneurial de Shapero et Sokol (1982) est généralement retenu pour analyser ce mode d'insertion (Audet, Riverin et Tremblay, 2005). Ce modèle explique l'intention entrepreneuriale par la désirabilité perçue et la faisabilité perçue de l'individu. La désirabilité est définie comme l'attrait ou l'attitude de l'individu vis-à-vis de l'entrepreneuriat. Elle est fonction du système de valeur de l'individu formé sous l'influence de son environnement culturel et social (la famille, les modèles d'entrepreneur, les groupes de pairs, le milieu scolaire, etc.). La faisabilité s'apparente à la croyance en l'existence et à l'accès aux compétences, ressources (financières, humaines et techniques) nécessaires, conditions facilitantes, etc. indispensables pour l'entame d'une création d'entreprise. Le capital culturel ainsi que le capital social de l'individu sont mobilisés à ce stade pour contrôler les variables liées à l'environnement (ressources, conditions facilitantes, rédaction d'un projet, etc.). 
La figure 1 illustre ce modèle.

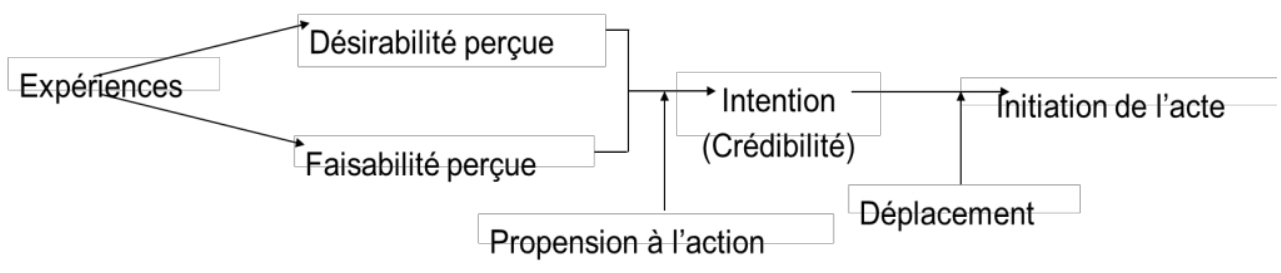

Figure 1 : Modèle de Shapero et Sokol d'après Krueger (1993)

Dans le modèle de Shapero et Sokol d'après Krueger (1993), les expériences de l'individu influencent la désirabilité et la faisabilité pour former la décision de créer une entreprise (intention entrepreneuriale). A partir de ce modèle, l'éducation à l'entrepreneuriat devrait agir aussi bien sur la désirabilité que sur la faisabilité. Une étude réalisée par Benié (2012b) en contexte de non enseignement de l'entrepreneuriat en Côte d'Ivoire a montré que les étudiants enquêtés avaient une faible intention entrepreneuriale en dépit d'une forte désirabilité entrepreneuriale. En revanche, leur perception de faisabilité était plutôt faible. Les répondants ont justifié cette faiblesse par l'absence d'une éducation à l'entrepreneuriat et à l'insatisfaction de certaines de leurs attentes relatives à leur formation. Les plus significatives sont entre autres "Etre capable de créer et gérer sa propre entreprise 》 et " Développer l'esprit d'entreprise ». Pour ces derniers, leur formation universitaire était trop théorique pour leur permettre de créer une entreprise au terme de leur formation académique. Ils ont préconisé en guise de solution des formations complémentaires pratiques axées sur l'entrepreneuriat dans leur domaine de formation.

Les écrits scientifiques mobilisés définissent l'insertion professionnelle comme le résultat de l'interaction entre le milieu scolaire, le milieu professionnel et l'acteur concerné. Ce résultat peut être évalué aussi bien au seuil d'entrée sur le marché du travail qu'après celui-ci. L'insertion sur le marché du travail possède deux modalités que sont le salariat et l'entrepreneuriat. Le salariat est représenté par les individus comme le modèle de travail de référence. Les stratégies d'insertion professionnelle mobilisées par les individus sont fondées sur leur capital social. La rareté et l'inégal accès aux emplois salariés vont positionner l'entrepreneuriat comme une alternative viable et socialement valorisante. Cette alternative permet à l'individu de minimiser la durée du chômage et de maximiser les chances d'insertion lors de sa transition sur le marché. L'insertion professionnelle par l'entrepreneuriat dépend, selon le modèle de Shapero et Sokol d'après Krueger (op. cit.), de la formation de l'intention entrepreneuriale et de déplacements survenus dans la vie de l'individu. L'éducation à l'entrepreneuriat agit particulièrement sur la faisabilité de l'acte 
entrepreneurial pour constituer l'intention de créer une entreprise. Pour ce faire, le programme de formation à l'entrepreneuriat dans les écoles doit prendre en compte les attentes exprimées par les apprenants et s'appliquer à les satisfaire. L'évaluation de l'implantation de l'éducation à l'entrepreneuriat est l'activité indiquée pour saisir les contraintes de l'environnement et les besoins nouveaux des apprenants. Les résultats de cette évaluation sont utiles pour réaliser les ajustements indispensables à l'atteinte des résultats escomptés par le programme d'éducation à l'entrepreneuriat. Or, l'implantation de l'entrepreneuriat comme une discipline officielle dans le programme des établissements de formation professionnelle n'a pas connu une telle activité d'évaluation. Les travaux de recherche sur l'éducation à l'entrepreneuriat en contexte de formation professionnelle sont quasi inexistants également. La présente étude vise donc à combler ce vide en analysant la pertinence de l'éducation à l'entrepreneuriat dans les établissements de formation professionnelle. De manière spécifique, il s'agit de décrire les attentes satisfaites par le cours d'entrepreneuriat chez les apprenants, de comparer les différents niveaux de satisfaction des apprenants en fonction de leur propension à entreprendre et d'expliquer la relation entre la satisfaction des attentes des apprenants relatives au cours d'entrepreneuriat et leur propension à entreprendre.

\section{Méthodologie}

Elle s'articule autour des rubriques que sont la population et l'échantillon de l'étude, l'instrument de collecte des données, le traitement et l'analyse des données.

\section{Population et échantillon}

La population d'enquête était composée de stagiaires finissants, inscrits en $3^{\text {ème }}$ année du Brevet de technicien (BT), dans les deux (2) lycées professionnels de la ville d'Abidjan que sont le lycée professionnel commercial de Yopougon (LPCY) et le lycée professionnel hôtelier de la Riviera (LPH). La technique d'échantillonnage systématique avait permis de sélectionner l'échantillon initial. Il était composé de $24,90 \%$ de 1 'ensemble des stagiaires du LPCY et de 31,10\% de 1'ensemble des stagiaires du LPH. Les données collectées auprès de ces répondants ont été modélisées à l'aide du modèle polytomique de $\operatorname{Rasch}^{6}$ afin d'obtenir une mesure de la

\footnotetext{
${ }^{6}$ L'intérêt pour le modèle de Rasch provient de sa simplicité et de sa capacité à positionner les sujets et les items sur une même échelle dans une logique de continuité (Van Alphen, Halfens, Hasman et Imbos, 1994). Les statistiques d'ajustement retenues sont l'outfit et l'infit dont le seuil est fixé à $\pm 2,5$. Ces statistiques sont utiles pour vérifier l'ajustement des données aux prescriptions du modèle de Rasch. Ils permettent donc de juger de la qualité de
} 
propension à entreprendre. Le modèle final obtenu au terme de la modélisation des données définit les caractéristiques de l'échantillon final dans le tableau 1 suivant.

Tableau 1 : Echantillon de l'étude

\begin{tabular}{ccccc}
\hline & Filières & Echantillon & Population mère & $\%$ \\
\hline \multirow{3}{*}{ LPCY } & Secrétariat bureautique & 35 & 161 & 21,74 \\
\cline { 2 - 5 } & Comptabilité & 26 & 181 & 14,36 \\
\cline { 2 - 5 } & Comptabilité-Commerce & 47 & 184 & 25,54 \\
\cline { 2 - 5 } & Total & 108 & 526 & 20,53 \\
\hline \multirow{3}{*}{ LPH } & Cuisine professionnelle & 21 & 184 & 11,41 \\
\cline { 2 - 5 } & Techniques hôtelières & 24 & 115 & 20,87 \\
\cline { 2 - 5 } & Total & 45 & 299 & 15,05 \\
\hline & Total général & 153 & 825 & 18,55 \\
\hline
\end{tabular}

L'échantillon, présenté dans le tableau 1, est composé de 18,50\% de l'ensemble des stagiaires de $3{ }^{\text {ème }}$ année BT des deux lycées professionnelles de la ville d'Abidjan. Il comporte $20,50 \%$ de l'ensemble des stagiaires du LPCY et de $15,05 \%$ de l'ensemble des stagiaires du LPH.

\section{Instrument de collecte des données}

L'instrument retenu est un questionnaire comportant des échelles de mesure de type Likert à 3 catégories de réponses allant de «Pas du tout d'accord» à «Tout à fait d'accord » pour la propension à entreprendre et de «Totalement satisfait» à " Totalement insatisfait» pour les attentes exprimées par les stagiaires. Les attentes des stagiaires relatives à leur formation en entrepreneuriat ont été collectées lors d'une enquête exploratoire réalisée auprès de stagiaires de 3 ème année BT du Centre de Perfectionnement aux Techniques Industrielles (CPTI) de Yopougon. Les items relatives à la propension à entreprendre ont été construits à partir du modèle de Shapero et Sokol d'après Krueger (1993) et validé avec un enseignant de la discipline "Entrepreneuriat» du CPTI. Le tableau 2 présente l'ensemble des items élaborés dans le cadre de cette étude et les variables visées.

l'ajustement et de détecter différents types de mauvais ajustement. Les hypothèses de base du modèle de Rash sont l'unidimensionnalité et l'indépendance locale. 
Tableau 2 : Présentation des variables et des items de l'étude

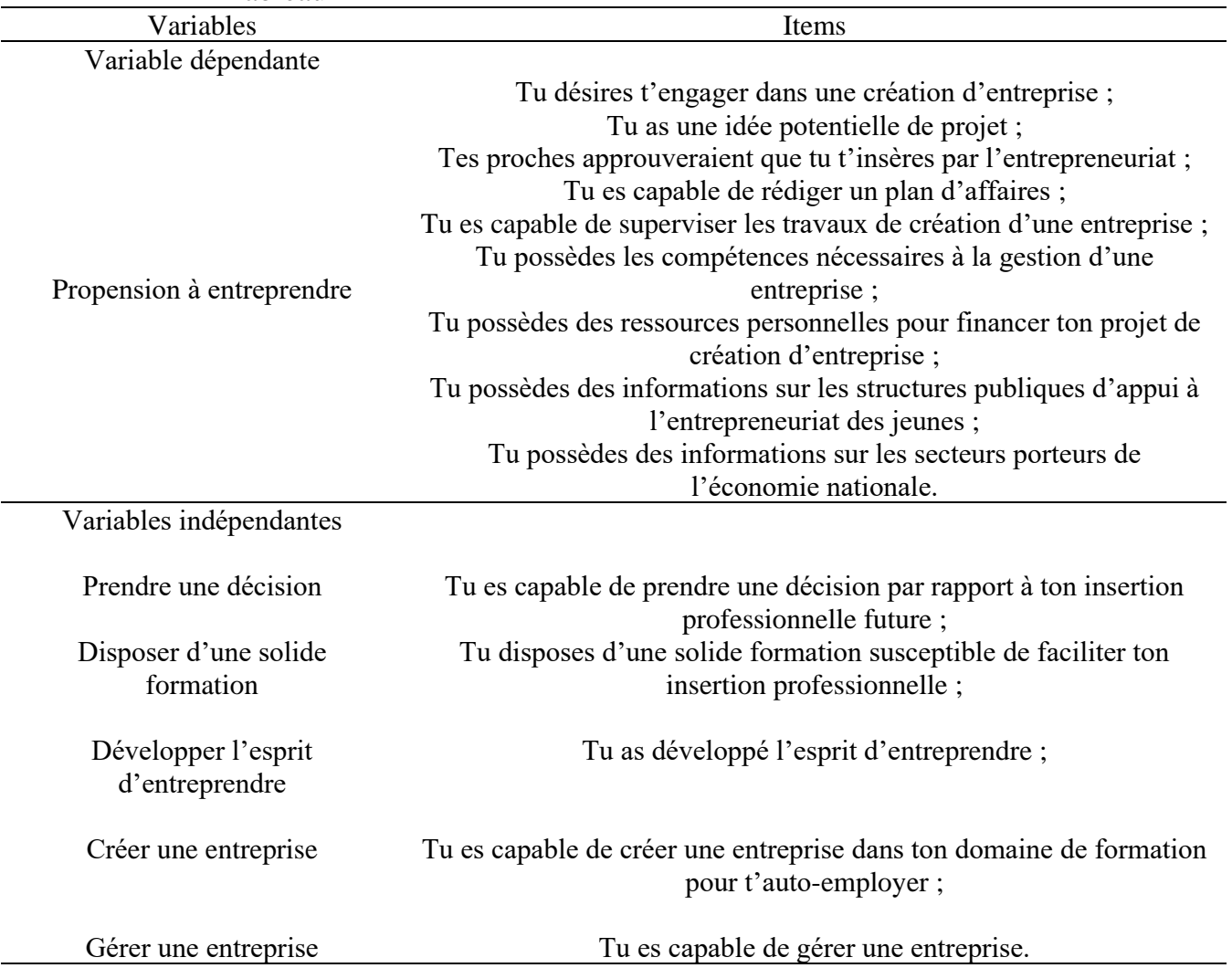

\section{Traitement et analyse des données}

Les sujets présentant des valeurs manquantes ont été retirés. Les données de l'enquête ont été saisies sous deux fichiers «Excel». Le fichier «Excel » relatif à la propension à entreprendre a été exporté vers le logiciel $\mathrm{R}$ pour la modélisation des données avec le modèle de Rasch. Ce traitement a été réalisé en mobilisant les librairies « $\mathrm{eRm}$ » et « data.table » du logiciel $\mathrm{R}$. Il a permis de retirer les sujets présentant un mauvais ajustement et de mesurer la propension à entreprendre des sujets restants sur une échelle continue. Les valeurs de cette mesure sont comprises entre -2 et 4.

Le fichier «Excel » relatif aux attentes exprimées par les stagiaires a été exporté vers le logiciel SPSS pour la réalisation de l'analyse descriptive. Cette analyse consiste en une comparaison de fréquences et de médianes. La médiane a été préférée à la moyenne car elle est plus robuste en présence de valeurs extrêmes.

Un troisième fichier «Excel », comportant les données relatives aux attentes exprimées par les stagiaires et la mesure de leur propension à entreprendre, a été constitué et exporté vers le logiciel SPSS pour l'analyse de régression multiple. Cette analyse vise à estimer la contribution relative 
de la satisfaction des différents types d'attentes exprimées par les stagiaires sur la variation de leur propension à entreprendre.

\section{Résultats}

La mesure de la propension à créer une entreprise chez les stagiaires des lycées professionnels de la ville d'Abidjan a été obtenue par la modélisation des données de l'enquête à l'aide du modèle polytomique de Rasch. Cette mesure est présentée en fonction du type de filières fréquentées par les stagiaires. Pour rappel, le lycée professionnel commercial de Yopougon dispense des formations dans des filières tertiaires et le lycée professionnel hôtelier dans des filières industrielles.

\section{Répartition de la propension à créer une entreprise chez les stagiaires}

La figure 2 ci-dessous donne la répartition de la propension à créer une entreprise chez les stagiaires en fonction du type de filière fréquenté.

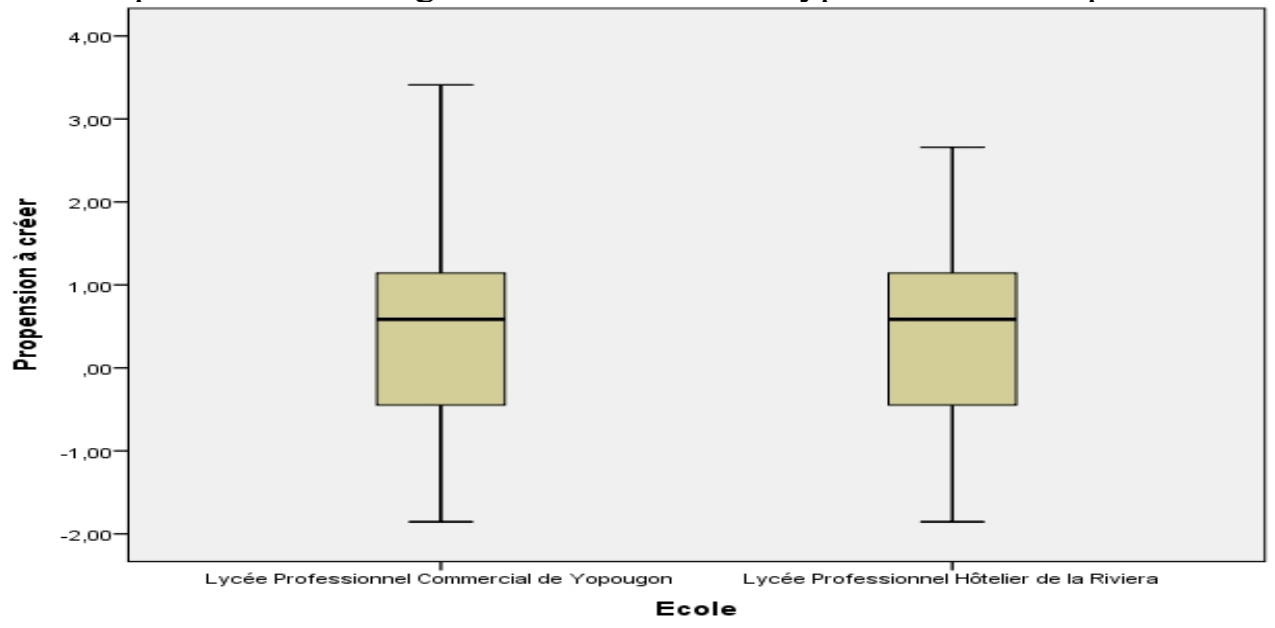

Figure 2 : Répartition de la propension des stagiaires à créer une entreprise en fonction de l'école fréquentée

La figure 2 montre que la propension à créer une entreprise chez les stagiaires de ces deux lycées professionnels est quasi identique. En effet, les trois premiers quartiles et la médiane des deux distributions coïncidentes quasiment. Ces distributions ne présentent pas de valeurs aberrantes.

\section{Satisfaction des attentes des stagiaires par le cours d'entrepreneuriat}

Cette satisfaction est appréhendée à l'aide des cinq items identifiés plus haut dans la méthodologie. La première attente est relative au développement de la capacité à prendre une décision par rapport à l'insertion professionnelle chez les répondants. 
Tableau 3 : Répartition des répondants en fonction de la satisfaction de leur attente relative à la prise de décision

\begin{tabular}{ccccc}
\hline & Fréquence & Pourcentage & $\begin{array}{c}\text { Pourcentage } \\
\text { valide }\end{array}$ & $\begin{array}{c}\text { Pourcentage } \\
\text { cumulé }\end{array}$ \\
\hline Totalement insatisfait & 26 & 17,0 & 17,0 & 17,0 \\
\hline Satisfait & 36 & 23,5 & 23,5 & 40,5 \\
\hline Totalement satisfait & 91 & 59,5 & 59,5 & 100,0 \\
\hline Total & $\mathbf{1 5 3}$ & $\mathbf{1 0 0 , 0}$ & $\mathbf{1 0 0 , 0}$ & \\
\hline
\end{tabular}

Le tableau 3 montre que 59,5\% des répondants estiment que le développement de leur capacité à prendre une décision par rapport à leur insertion professionnelle future est totalement satisfaisant.

Tableau 4 : Répartition des répondants en fonction de la satisfaction de leur attente relative à la disposition d'une formation solide

\begin{tabular}{ccccc}
\hline & Fréquence & Pourcentage & $\begin{array}{c}\text { Pourcentage } \\
\text { valide }\end{array}$ & $\begin{array}{c}\text { Pourcentage } \\
\text { cumulé }\end{array}$ \\
\hline Totalement insatisfait & 24 & 15,7 & 15,7 & 15,7 \\
\hline Satisfait & 48 & 31,4 & 31,4 & 47,1 \\
\hline Totalement satisfait & 81 & 52,9 & 52,9 & 100,0 \\
\hline Total & $\mathbf{1 5 3}$ & $\mathbf{1 0 0 , 0}$ & $\mathbf{1 0 0 , 0}$ & \\
\hline
\end{tabular}

Les résultats du tableau 4 montrent que 52,9\% des répondants estiment que le cours d'entrepreneuriat a répondu de manière totalement satisfaisante à leur attente qui consiste à disposer d'une solide formation susceptible de faciliter leur insertion professionnelle.

Tableau 5 : Répartition des répondants en fonction de la satisfaction de leur attente relative au développement de l'esprit d'entreprendre

\begin{tabular}{ccccc}
\hline & Fréquence & Pourcentage & $\begin{array}{c}\text { Pourcentage } \\
\text { valide }\end{array}$ & $\begin{array}{c}\text { Pourcentage } \\
\text { cumulé }\end{array}$ \\
\hline Totalement insatisfait & 12 & 7,8 & 7,8 & 7,8 \\
\hline Satisfait & 42 & 27,5 & 27,5 & 35,3 \\
\hline Totalement satisfait & 99 & 64,7 & 64,7 & 100,0 \\
\hline Total & $\mathbf{1 5 3}$ & $\mathbf{1 0 0 , 0}$ & $\mathbf{1 0 0 , 0}$ & \\
\hline
\end{tabular}

Le tableau 5 montre que le cours d'entrepreneuriat a répondu de manière totalement satisfaisante à l'attente relative au développement de l'esprit d'entreprendre de $64,7 \%$ des répondants.

Tableau 6 : Répartition des répondants en fonction de la satisfaction de leur attente relative à la capacité de créer sa propre entreprise

\begin{tabular}{ccccc}
\hline & Fréquence & Pourcentage & $\begin{array}{c}\text { Pourcentage } \\
\text { valide }\end{array}$ & $\begin{array}{c}\text { Pourcentage } \\
\text { cumulé }\end{array}$ \\
\hline Totalement insatisfait & 21 & 13,7 & 13,7 & 13,7 \\
\hline Satisfait & 45 & 29,4 & 29,4 & 43,1 \\
\hline Totalement satisfait & 87 & 56,9 & 56,9 & 100,0 \\
\hline Total & $\mathbf{1 5 3}$ & $\mathbf{1 0 0 , 0}$ & $\mathbf{1 0 0 , 0}$ & \\
\hline
\end{tabular}


Le tableau 6 montre que 56,9\% des répondants estiment que le cours d'entrepreneuriat a répondu de manière totalement satisfaisante à leur attente relative au développement de leur capacité à créer une entreprise dans leur domaine de formation pour s'auto-employer.

Tableau 7 : Répartition des répondants en fonction de la satisfaction de leur attente relative à la capacité de gérer leur propre entreprise

\begin{tabular}{ccccc}
\hline & Fréquence & Pourcentage & $\begin{array}{c}\text { Pourcentage } \\
\text { valide }\end{array}$ & $\begin{array}{c}\text { Pourcentage } \\
\text { cumulé }\end{array}$ \\
\hline Totalement insatisfait & 19 & 12,4 & 12,4 & 12,4 \\
\hline Satisfait & 50 & 32,7 & 32,7 & 45,1 \\
\hline Totalement satisfait & 84 & 54,9 & 54,9 & 100,0 \\
\hline Total & $\mathbf{1 5 3}$ & $\mathbf{1 0 0 , 0}$ & $\mathbf{1 0 0 , 0}$ & \\
\hline
\end{tabular}

Le tableau 7 montre que le cours d'entrepreneuriat a répondu de manière totalement satisfaisante à l'attente relative au développement de la capacité à gérer une entreprise chez $64,7 \%$ des répondants.

\section{Attente satisfaite par le cours d'entrepreneuriat et propension à créer une entreprise}

Cette rubrique est consacrée à l'analyse de la relation entre la propension à créer une entreprise et chacune des attentes satisfaites par le cours d'entrepreneuriat.

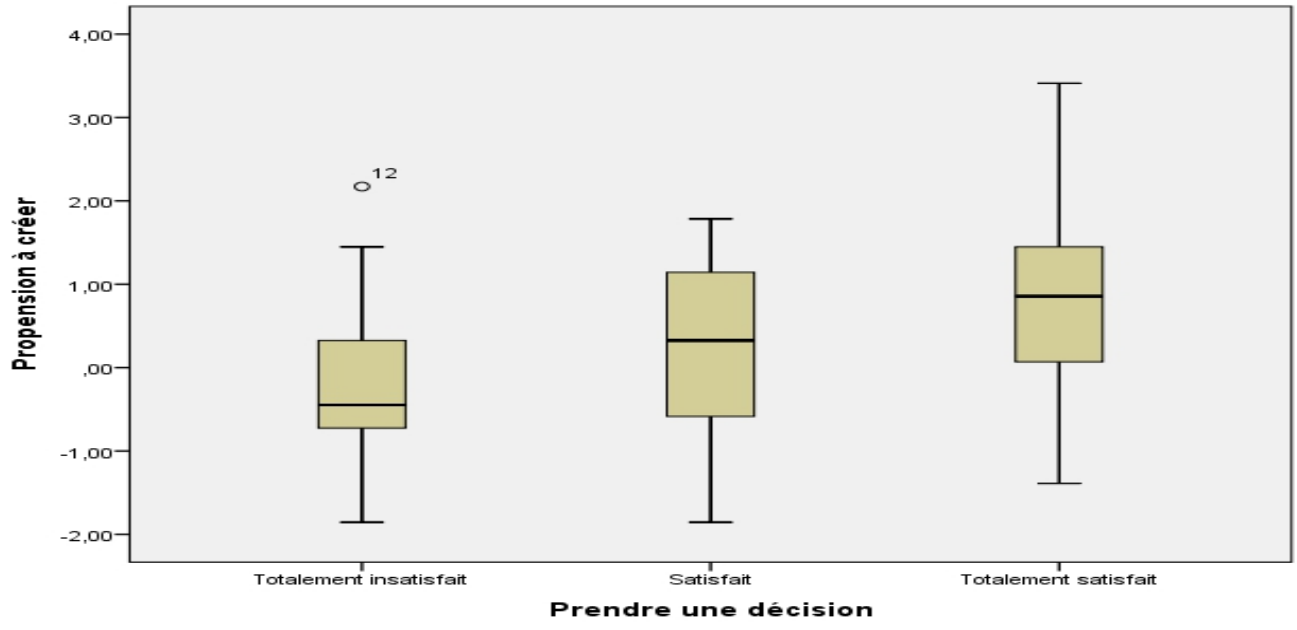

Figure 3 : Relation entre le développement de la capacité à prendre une décision et la propension à créer une entreprise

La figure 3 montre qu'il existe une relation monotone croissante entre le développement de la capacité à prendre une décision chez les répondants en vue d'une insertion professionnelle future et leur propension à créer une entreprise. En effet, plus l'attente est jugée satisfaisante, plus la médiane de la propension à entreprendre est grande. 


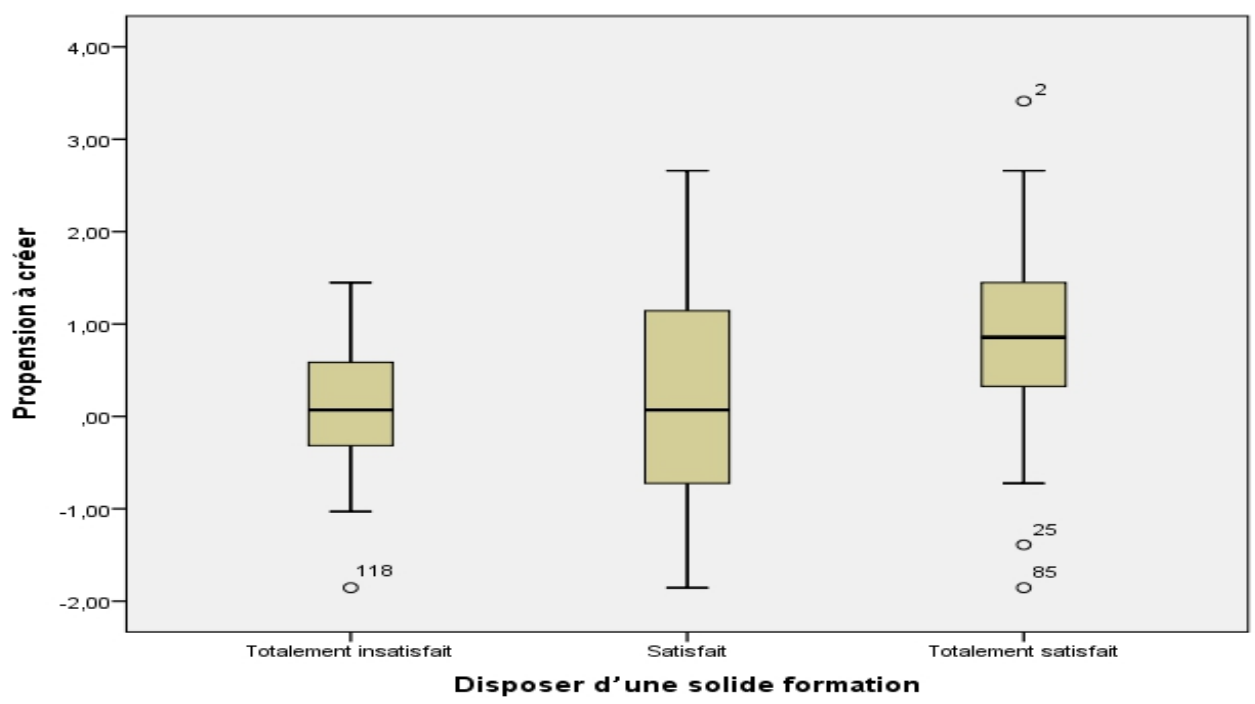

Figure 4 : Relation entre la possession d'une solide formation en vue du salariat et la propension à créer une entreprise

La figure 4 montre qu'il existe une relation monotone croissante entre le fait de disposer une solide formation en vue d'une insertion par le salariat et la propension à créer une entreprise. Cependant, la distribution de la propension à créer une entreprise chez les répondants en fonction de leur satisfaction ont quasiment la même médiane pour les appréciations «totalement insatisfait» et «satisfait». En plus, la figure met en exergue quatre valeurs extrêmes ou atypiques dont trois pour l'appréciation libellée « totalement satisfaisant $»$.

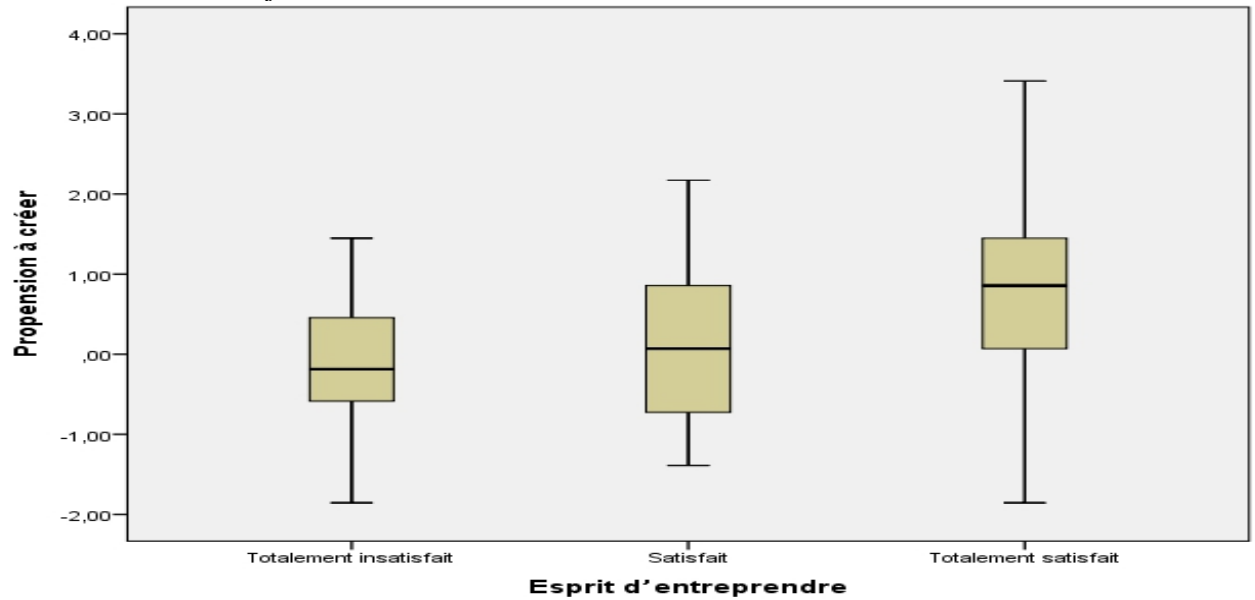

Figure 5 : Relation entre le développement de l'esprit d'entreprendre et la propension à créer une entreprise 
La figure 5 montre qu'il existe une relation monotone croissante entre le développement de l'esprit d'entreprendre chez les répondants et leur propension à créer une entreprise. L'intervalle interquartile de l'appréciation «totalement satisfait» est concentré par rapport au reste de la distribution. Cependant, les frontières hautes et basses de la moustache sont étalées par rapport à la boîte.

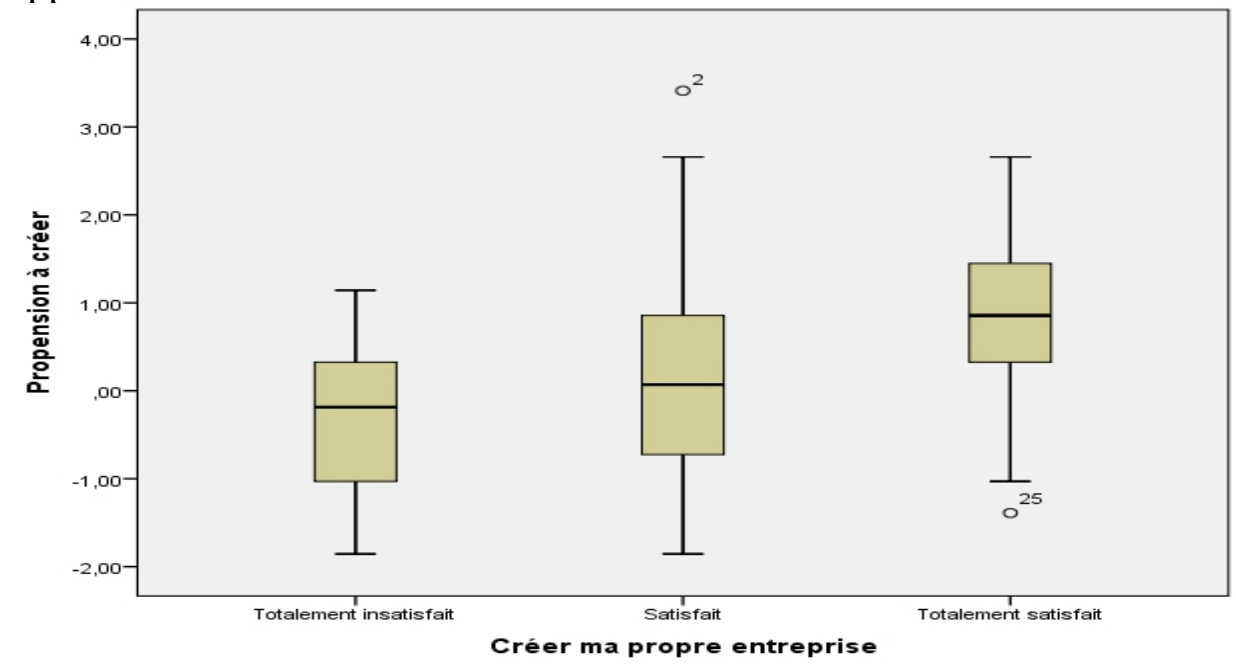

Figure 6 : Relation entre le développement de la capacité de créer sa propre entreprise et la propension à créer une entreprise

La figure 6 montre qu'il existe une relation monotone croissante entre le développement de l'esprit d'entreprendre chez les répondants et leur propension à créer une entreprise. La boîte à moustache de l'appréciation «totalement satisfait » est quasi symétrique.

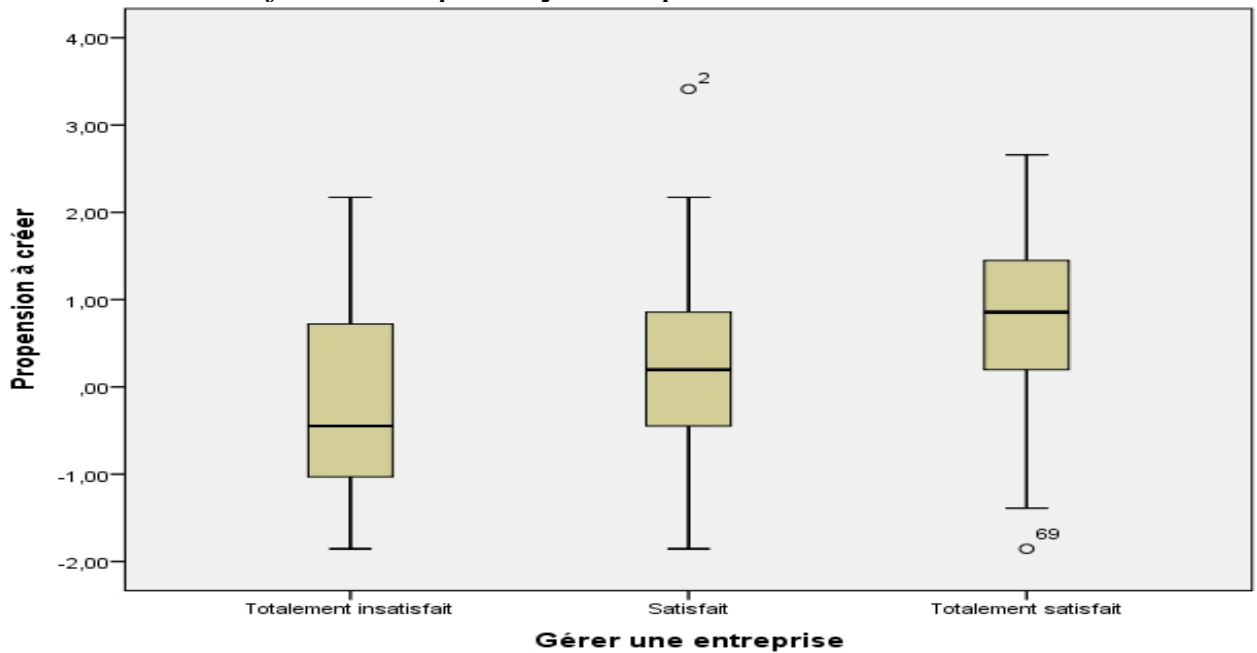

Figure 7 : Relation entre le développement de la capacité de gérer une entreprise et la propension à créer une entreprise 
La figure 7 montre qu'il existe une relation monotone croissante entre le développement de l'esprit d'entreprendre chez les répondants et leur propension à créer une entreprise. Cependant, les distributions de la propension à entreprendre des trois boîtes à moustaches ont quasiment la même étendue.

\section{Influence de la satisfaction des attentes des stagiaires sur leur propension à entreprendre}

Les analyses réalisées au cours des rubriques précédentes ont montré l'existence d'une relation monotone croissante entre la satisfaction des différents types d'attentes exprimées par les stagiaires et leur propension à entreprendre. La présente rubrique va chercher à estimer la contribution relative de chacune de ces attentes sur la variation de la propension à entreprendre des stagiaires afin d'isoler les plus significatives.

Tableau 8: Analyses de régression du modèle de propension entrepreneuriale

\begin{tabular}{|c|c|c|c|c|c|}
\hline \multirow[t]{2}{*}{ Modèle } & \multicolumn{2}{|c|}{$\begin{array}{l}\text { Coefficients } \\
\text { Non standardisés }\end{array}$} & \multirow{2}{*}{$\begin{array}{c}\text { Coefficients } \\
\text { standardisés } \\
\text { Beta } \\
\end{array}$} & \multirow[t]{2}{*}{$\mathrm{t}$} & \multirow[t]{2}{*}{ Sig. } \\
\hline & $\mathrm{B}$ & Erreur Std. & & & \\
\hline (Constant) & $-1,274$ & 0,249 & & $-5,114$ & 0,000 \\
\hline Créer ma propre entreprise & 0,407 & 0,118 & 0,280 & 3,440 & 0,001 \\
\hline Gérer une entreprise & 0,060 & 0,127 & 0,040 & ,469 & 0,640 \\
\hline Prendre une décision & 0,287 & 0,104 & 0,209 & 2,751 & 0,007 \\
\hline $\begin{array}{c}\text { Développer l'esprit } \\
\text { d'entreprendre }\end{array}$ & 0,277 & 0,125 & 0,167 & 2,224 & 0,028 \\
\hline $\begin{array}{c}\text { Disposer d'une solide } \\
\text { formation }\end{array}$ & 0,182 & 0,105 & 0,128 & 1,741 & 0,084 \\
\hline $\begin{array}{c}\mathrm{R} \\
\mathrm{R}^{2}\end{array}$ & & & 0,541 & & \\
\hline
\end{tabular}

Les analyses de régression, contenues dans le tableau 8, montrent que $29,2 \%\left(\mathrm{R}^{2}=0,292\right)$ de la propension entrepreneuriale des stagiaires répondants peuvent être expliqués par la satisfaction des attentes que sont, dans l'ordre décroissant, "Créer ma propre entreprise», "Prendre une décision» et "Esprit d'entreprendre» au seuil de 0,05. Ces résultats confirment l'existence d'une relation monotone croissante significative entre la propension entrepreneuriale des stagiaires et la satisfaction de trois attentes que sont "Créer ma propre entreprise », "Prendre une décision » et «Esprit d'entreprendre». L'attente relative au développement des capacités indispensables à la création d'une entreprise afin de s'auto employer s'avère une variable déterminante (la valeur $t=3,440$ et le degré de signification sig. $=0,001$ ) de la propension entrepreneuriale.

\section{Pertinence de l'éducation à l'entrepreneuriat}

Les résultats de l'étude montrent que la distribution de la propension à entreprendre des stagiaires du lycée professionnel commercial de 
Yopougon est quasi identique à celle des stagiaires du lycée professionnel hôtelier de la Riviera. Ce résultat peut s'expliquer par la formation initiale des formateurs en entrepreneuriat dans le même institut pédagogique et l'usage du même programme de formation en entrepreneuriat dans les établissements de formation professionnelle. Mais, les stagiaires des deux établissements estiment, dans une grande proportion, que la formation en entrepreneuriat n'a pas répondu de manière totalement satisfaisante à leurs attentes. En effet, cet avis est partagé par $40,50 \%$ des stagiaires pour l'attente "Prendre une décision relative à l'insertion professionnelle future", $47,10 \%$ pour l'attente "Disposer d'une solide formation susceptible de faciliter l'insertion professionnelle », 35,30\% pour l'attente "Développer l'esprit d'entreprendre », 43,10\% pour l'attente "Créer une entreprise dans son domaine de formation pour s'auto employer », et 35,30\% pour l'attente «Gérer une entreprise ». Ces proportions, supérieures à 35\%, montrent que l'éducation à l'entrepreneuriat dans les lycées professionnels de la ville d'Abidjan semble ne pas être pertinente pour un nombre important de stagiaires. Ceci peut s'expliquer par le caractère plutôt théorique de cette éducation à l'entrepreneuriat notamment l'absence d'activités pédagogiques telles que les microentreprises ou les pépinières d'entreprise. A ce niveau, l'étude s'interroge sur la relation entre la satisfaction des attentes exprimées par les stagiaires et leur propension à entreprendre. L'analyse des boîtes à moustaches a montré une relation plutôt monotone croissante entre la satisfaction des attentes des stagiaires et leur propension à entreprendre. L'analyse du modèle de régression multiple de la propension à entreprendre a montré, quant à elle, une influence significative par ordre d'importance des attentes "Créer une entreprise dans son domaine de formation pour s'auto employer ", "Prendre une décision relative à l'insertion professionnelle future » et "Développer l'esprit d'entreprendre ». Ces résultats montrent que les attentes qui sont endossées par une proportion élevée de stagiaires totalement satisfaits ne sont pas celles qui ont une plus grande influence significative. Ceci traduit l'effet (pertinence en fin de formation) de l'éducation à l'entrepreneuriat sur la propension à entreprendre notamment par la satisfaction des attentes que sont "Créer une entreprise dans son domaine de formation pour s'auto-employer", "Prendre une décision relative à l'insertion professionnelle future» et "Développer l'esprit d'entreprendre ».

\section{Discussion}

Les résultats de l'étude montrent que l'insertion par l'entrepreneuriat est envisagée par les apprenants comme une alternative viable pour construire une carrière professionnelle. L'éducation à l'entrepreneuriat s'avère être une stratégie efficace de construction sociale du modèle 
d'insertion professionnelle par l'entrepreneuriat. Cette éducation structure la désirabilité et la faisabilité de l'acte entrepreneurial au travers d'un processus de socialisation qui tienne compte des besoins des apprenants et des contraintes du marché du travail. Ces résultats confirment ceux de Benié (2012b) obtenus en contexte de non enseignement de l'entrepreneuriat dans les universités de Cocody et d'Abobo-Adjamé. Les attentes relatives à l'éducation à l'entrepreneuriat exprimées par la majorité des étudiants étaient "Etre capable de créer et gérer sa propre entreprise 》 et "Développer l'esprit d'entreprise ». Ces résultats convergent également avec ceux des auteurs tels que Pepin (2011a), Boissin, Chollet et Emin (2005), Alves (2005), Kallel (2005) ou encore Verstraete (1998). Ces auteurs montrent que l'éducation à l'entrepreneuriat a une influence significative sur l'attitude des apprenants vis-à-vis de l'entrepreneuriat. Les résultats de l'étude positionne l'éducation à l'entrepreneuriat comme un puissant outil de sensibilisation à l'alternative de carrière professionnelle que constitue l'entrepreneuriat. Ces résultats ne peuvent cependant pas être généralisés à l'ensemble du système de formation professionnelle même si l'étude porte à la fois sur des filières tertiaires et des filières industrielles. Les futurs travaux devront donc prendre en compte un échantillon plus grand d'établissement de formation professionnelle. Ces travaux doivent s'appliquer à analyser la pertinence du programme de formation à l'entrepreneuriat mis en œuvre dans les établissements, l'influence des caractéristiques sociodémographiques des stagiaires de l'ETFP sur leur propension à entreprendre ainsi que les trajectoires d'insertion par l'entrepreneuriat des $09,78 \%$ des stagiaires de 2016 qui ont réussi à s'insérer par ce mode.

\section{References:}

1. Aka, A. (2010). Comment le monde moderne contribue t- il à l'insertion des jeunes. Dans K. Koffi (dir.). Problématique de l'insertion socio-professionnelle des jeunes en Côte d'Ivoire. Actes du colloque. Abidjan : Centre de Recherche et d'Action pour la Paix (CERAP), pp 61 - 74.

2. Alves, M. G. (2005). Perspective éducative sur l'insertion professionnelle des diplômés de l'enseignement supérieur. Revue européenne de formation professionnelle, 34 , pp. 30 - 43.

3. Audet, J. ; Riverin, N. \& Tremblay, M. (2005). L'influence de la culture d'un pays sur la propension entrepreneuriale de ses citoyens : Le cas du Canada. Cahier de recherche, 12, pp 1 - 16. Repéré à http://expertise.hec.ca/chaire_entrepreneuriat/wp-content/uploads/2005-12icppecitoyens.pdf.

4. Bazin, L. \& Yao Gnabeli, R. (1997). Le travail salarié en Côte d'Ivoire, un modèle en décomposition ? » Dans H. Memel-Foté \& B. 
Contamin (dir.). Le modèle ivoirien en questions. Crises, ajustements, recompositions, Paris : Karthala-ORSTOM, 1997, pp 689-705.

5. Benié, A. (2012a). Formation universitaire et développement de l'intention de créer une entreprise chez les étudiants. Thèse unique de Doctorat de Sociologie de l'éducation, spécialité développement du capital humain, Université Félix Houphouët Boigny de Cocody, Abidjan, Côte d'Ivoire.

6. Benié, A. (2012b). Attentes relatives à la formation initiale et choix professionnel chez les étudiants. African Education Development Issues (AEDI), Afreducdev issues ISSN 2079-651X, ROCARE $\mathrm{N}^{\circ} 4$. Spécial JRECI 2011.

7. Boissin, J. Chollet, B. \& Emin, S. (2005). Les croyances des étudiants envers la création d'entreprise : un état des lieux. Universités de Grenoble, France.

8. Brou, F. R. (2016). Problématique de l'insertion socioprofessionnelle des jeunes diplômés des universités publiques de Côte d'Ivoire. Thèse unique de Doctorat de Sociologie de l'économie et de l'emploi, Université Félix Houphouët Boigny de Cocody, Abidjan, Côte d'Ivoire.

9. Bruyat, C. (1993). Création d'entreprise : contributions épistémologiques et Modélisation. Thèse de doctorat en sciences de gestion, Université Pierre Mendès, Grenoble, France.

10. Cros, F. (2010). Introduction: objectifs et apports du séminaire. Dans V. Tehio (dir.). Politiques publiques en éducation : l'exemple des réformes curriculaires. Actes du séminaire final de l'étude sur les réformes curriculaires par l'approche par compétences en Afrique. Repéré à http://www.ciep.fr/sites/default/files/migration/publi_educ/docs/actesreformes-curriculaires.pdf.

11. Diro, T. (2010). Etat des lieux. Dans K. Koffi (dir.). Problématique de l'insertion socio-professionnelle des jeunes en Côte d'Ivoire. Actes du colloque. Abidjan : Centre de Recherche et d'Action pour la Paix (CERAP), pp 21 - 34.

12. Drolet, J-L. Monette, M. \& Pelletier, R. (1996). Un regard dialectique sur l'expérience de transition études-travail. Revue des sciences de l'éducation, 22 (1), p. 97-118. Repéré à http://id.erudit.org/iderudit/031848ar.

13. Fonds Africain de Développement (2013). Côte d'Ivoire : Programme d'Appui à l'Amélioration de l'Employabilité et de l'Insertion des Jeunes (PAAEIJ) (Rapport d'évaluation). Repéré à https://www.afdb.org/fileadmin/uploads/afdb/Documents/Côte_d_ Ivoire_- 
_Programme_d'appui_à_l'amélioration_de_l'employabilité_et_de_l' insertion_des_jeunes_PAAEIJ___Rapport_d_évaluation.pdf.

14. Fortino, S. (2009). L'apport des trajectoires sociales pour comprendre la précarité en féminin. L'exemple d'une recherche sur l'insertion professionnelle de chômeuses de longue durée. Papeles del CEIC, 1 , pp. 1 - $30 . \quad$ Repéré à http://www.identidadcolectiva.es/pdf/44.pdf.

15. Gartner, W. (1988). Who is an entrepreneur?" is the wrong question. American Journal of Small Business, 12 (4), pp. 11-32.

16. Gérard, F.-M. (2003). L'évaluation de l'efficacité d'une formation, Gestion 2000, 20 (3), pp. 13-33.

17. Gnabeli, Y. \& Lognon, J-L. (2010). Construction sociale des monopoles dans l'économie informelle abidjanaise. Itineris Plus, 8 (8), $15 \mathrm{p}$.

18. Kallel, T. (2005). Etats des lieux de l'enseignement de l'entrepreneuriat dans les Universités tunisiennes : Pour une évaluation des couples enseignements/établissements dans leur capacité à créer une dynamique entrepreneuriale. Communication présentée au $4^{\text {ème }}$ congrès de l'Académie de l'entrepreneuriat, Paris, France.

19. Kane, D. O. ; Sy, T. ; Massing, N. P. \& Liboudou, L. (2014). Les Déterminants de l'Entrepreneuriat des Jeunes en Afrique de l'Ouest: Le Cas de la Mauritanie et du Sénégal (Rapport de Recherche du FRCIEA No 81/14). Dakar, Sénégal : Fonds de Recherche sur le Climat d'Investissement et l'Environnement des Affaires.

20. Kolvereid, I. (1996). Prediction of employment status choice intentions. Entrepreneurship Theory and Practice, pp. 47 - 56.

21. Koné, D. (2010). Développement local, une préoccupation des élus locaux ? Les collectivités territoriales aujourd'hui dans le Nord de la Côte d'Ivoire face aux enjeux de l'insertion socio-professionnelle des jeunes comme stratégie de promotion humaine. Dans K. Koffi (dir.). Problématique de l'insertion socio-professionnelle des jeunes en Côte d'Ivoire. Actes du colloque. Abidjan : Centre de Recherche et d'Action pour la Paix (CERAP), pp 145 - 159.

22. Koné, K., Koffi，K. \& Ehui, P. (2016). Jeunes et insertion professionnelle en Côte d'Ivoire : la formation professionnelle à l'épreuve des pratiques d'insertion. Revue Internationale des Sciences et Technologies de l'Education, ${ }^{\circ}$ 6, p. $75-110$.

23. Kouadio, B. (1998). L'impact des programmes d'ajustement structurel sur le secteur formel en Côte d'Ivoire : une analyse différenciée selon le genre (Rapport $\mathrm{n}^{\circ}$ MFM 552). Abidjan, Côte d'Ivoire : Organisation international du travail (OIT). 
24. Krueger, N. (1993). The impact of prior entrepreneurial exposure on perceptions of new venture feasability and desirability. Entrepreneurship Theory \& Practice, pp. 5-20.

25. Laflamme, C. (1984) "Une contribution à un cadre théorique sur l'insertion professionnelle des jeunes." Revue des sciences de l'éducation, 102, pp. 199-216.

26. Laviolette, E., \& Loue, C. (2006). Les compétences entrepreneuriales : définition et construction d'un référentiel. Repéré à

https://jecreedansmaregion.fr/sites/default/files/projet360/les_competences_entrepreneuriales__definition_et_construction_dun_referentiel__propose_par_mm._laviolette_et_loue.pdf.

27. Lévy-Tadjine, T., Chelly, A., \&Paturel, R. (2006). Pour déconstruire le concept d'entrepreneuriat institutionnel et ses utilisations abusives en Management Stratégique. Communication présentée au $8^{\text {è }}$ congrès international francophone en entrepreneuriat et PME, Fribourg, Suisse.

28. Ministère d'Etat, Ministère de l'Emploi, des Affaires Sociales et de la Formation Professionnelle (2014a). Politiques et dispositifs d'insertion professionnelle et de création d'emplois : cas de la Côte d'Ivoire. Abidjan, Côte d'Ivoire. Repéré à http://www.adeanet.org/pqipdctp/sites/default/files/documents/rapport_pays_cote_divoire.pdf.

29. Ministère d'Etat, Ministère de l'Emploi, des Affaires Sociales et de la Formation Professionnelle (2014b). Enquête nationale sur la situation de l'emploi Et du travail des enfants (ENSETE 2013). Rapport descriptif sur la situation de l'emploi. Abidjan, Côte d'Ivoire.

Repéré http://www.ins.ci/n/documents/travail_enfant/Enqute\%20nationale\%202013.pdf.

30. OIT \& PNUD (2000). "Des Emplois pour l'Afrique ": stratégie d'Emploi aptes à réduire la Pauvreté en Afrique. Plan d'action national. Abidjan, Côte d'Ivoire.

31. Parmentier, P. R. (2015). De l'idée au projet : les parcours des créateurs d'entreprise jeunes et seniors. Thèse de doctorat de Sociologie, université Paris 8, France. Repéré à www.theses.fr/2015PA080039.pdf.

32. Pepin, M. (2011a). L'éducation entrepreneuriale au primaire et au secondaire : Gros plan sur la micro-entreprise scolaire. Revue canadienne de l'éducation, $\mathrm{n}^{\circ} 34$, pp 280 - 300.

33. Pepin, M. (2011b). L'entrepreneuriat en milieu scolaire : de quoi s'agit-il? McGill Journal of Education, 46 (2), pp. 303 - 326. DOI : 10.7202/1006441ar. 
34. Plante J. \& Bouchard C. (1998). La qualité. Sa définition et sa mesure. Service social, 47 (1-2), pp. 27-61.

35. Regroupement des acteurs ivoiriens des droits humains (2014). Côte d'Ivoire: une décennie de crimes non encore punis. Repéré à https://freedomhouse.org/sites/default/files/Cote\%20dIvoire\%20report.pdf.

36. Schumpeter, J. (1935). La théorie de l'évolution économique, recherches sur le profit, l'intérêt et le cycle de la conjoncture. Paris : Dalloz.

37. Secrétariat d'Etat chargé de l'enseignement technique et de la formation professionnelle (2017). Mise en place d'un système de suivi des diplômés de l'ETFP et calcul du taux d'insertion des diplômés de l'année 2016. Abidjan, Côte d'Ivoire: Direction de la Formation Qualifiante, de l'Apprentissage et de l'Insertion.

38. Shane, S. \& Venkataraman, S. (2000). The Promise of entrepreneurship as a field of Research. Academy of Management Review, 1 (25), pp. 217-226.

39. Shapero, A. \& Sokol, L. (1982). The social dimension of entrepreneurship. In The encyclopedia of entrepreneurship. Kent, C, Sexton, D., \& Vesper, K. (dir.). Englewood Cliffs NJ, pp. 72-90.

40. Tounés, A. (2006). "L'intention entrepreneuriale des étudiants : le cas français ". La Revue des Sciences de Gestion, n²19, p. 57-65. DOI 10.3917/rsg.219.0057.

41. Trottier, C. (2000). Questionnement sur l'insertion professionnelle des jeunes. Lien social et politiques, 43, pp. 93-101. Repéré à http://id.erudit.org/iderudit/005242ar.

42. Van Alphen, A. ; Halfens, R. ; Hasman A. \& Imbos, T. (1994). Likert or Rasch? Nothing is more applicable than good theory. Journal af Advanced Nursing, vol. 20, p. 196-201.

43. Vernières, M. (1997). La notion d'insertion professionnelle. Dans M. Vernières (dir.). L'insertion professionnelle : analyses et débats, pp 9 - 22. Paris : Economica.

44. Verstraete, T. \& Fayolle, A. (2005). Paradigmes et entrepreneuriat. Revue de l'entrepreneuriat, 4 (1), pp. 33 - 52.

45. Verstraete, T. (1998). Esprit entrepreneurial et cartographie cognitive: utilisations académiques, pratiques et pédagogique de l'outil. Communication présentée au congrès Enseignement supérieur et PME. Repéré à http://asso.nordnet.fr/ae/TV0398.pdf. 